\title{
Swift heavy ion damage to sodium chloride: synergy between excitation and thermal spikes
}

\author{
A Rivera , J Olivares , G Garcia and F Agulló-López
}

\begin{abstract}
Systematic data on the effect of irradiation with swift ions ( $\mathrm{Zn}$ at $735 \mathrm{MeV}$ and Xe at $929 \mathrm{MeV}$ ) on $\mathrm{NaCl}$ single crystals have been analysed in terms of a synergetic two-spike approach (thermal and excitation spikes). The coupling of the two spikes, simultaneously generated by the irradiation, contributes to the operation of a non-radiative exciton decay model as proposed for purely ionization damage. Using this scheme, we have accounted for the $\pi$-emission yield of self-trapped excitons and its temperature dependence under ion-beam irradiation. Moreover, the initial production rates of F-centre growth have also been reasonably simulated for irradiation at low temperatures $(<100 \mathrm{~K})$, where colour centre annealing and aggregation can be neglected.
\end{abstract}

\section{Introduction}

Dielectric crystals can be heavily damaged by high-energy ions. They deposit energy by two main mechanisms, elastic nuclear collisions and electronic excitations, that are respectively associated to the nuclear, $S_{\mathrm{n}}$, and electronic, $S_{\mathrm{e}}$, stopping powers (energy deposited per ion and unit depth). The damage caused by elastic collisions is reasonably well understood and simulation codes are available to describe it $[1,2]$. On the other hand, our understanding of the damage due to electronic excitations is still poor [3] and moreover, appears to be strongly dependent on the particular material under irradiation due to aspects as the type of bonding or the energy band gap. For swift heavy ions, i.e. those having mass number $A>15$ and energies around or above $0.1 \mathrm{MeV} \mathrm{amu}^{-1}, S_{\mathrm{e}}$ is greatly dominant over $S_{\mathrm{n}}$, which results in a challenge for materials scientists to describe the damage phenomena. Several proposals have been advanced to deal with this problem such as Coulomb explosion $[4,5]$, thermal spike [6-8] and excitonic models [3, 9-12].

So far, the thermal spike model has offered a satisfactory explanation for several relevant features of the ion-beam damage by electronic excitation, such as the formation of amorphous tracks in crystals along the ion trajectories. In particular, it provides a clear explanation for the occurrence of a well-defined stopping power threshold to generate the tracks (thresholding effect). However, this model cannot account for other features such as the formation of point defects, the cumulative character of the damage and the sub-threshold damage, unless some additional processes for defect production are taken into account. In order to describe these other features of damage a new model $[10,11]$ has been recently developed for $\mathrm{LiNbO}_{3}$, based on the non-radiative decay of localized (e.g. self-trapped) excitons. The model, 
initially suggested by Itoh [9], relies on a synergy between the excitation and thermal spikes, thus we refer to it as the two-spike model. It explains satisfactorily the nonlinear dependence of the damage with electronic stopping power, the thresholding effect for amorphization and the cumulative character of damage. Unfortunately, the model for this crystal had to be maintained at a phenomenological level because significant spectroscopic information on exciton formation and dynamics in $\mathrm{LiNbO}_{3}$ is still lacking.

Alkali halides are highly ionic dielectric crystals with simple (mostly fcc) crystal structures with large band gap that are easily damaged by purely ionizing irradiation (UV light, x-rays or electrons). For these crystals a model based on the non-radiative decay of self-trapped excitons has been well documented for many years [3, 13-15] and satisfactorily accounts for damage evolution upon ionizing radiation. Therefore, alkali halides constitute ideal candidates to test our model based on the synergy between the two spikes (thermal and excitation spikes) during ion-beam bombardment, as proposed for $\mathrm{LiNbO}_{3}$. In fact, the self-trapping of excitons and their radiative and non-radiative decay as a function of temperature have been thoroughly investigated [3, 15-18]. Moreover, some experiments have been so far reported on swift-ion damage to alkali halides focusing on the dynamics of the thermal spike following ion impact [19-23].

The purpose of this paper is to apply the synergetic twospike model $[10,11]$ to some careful experimental data taken by the GANIL group on $\mathrm{NaCl},[19-21]$, where experimental details can be consulted. This crystal is particularly adequate since it shows a neat anticorrelation between the $\pi$ luminescence emission and the F-centre production rate. Although some simulations for the luminescence yield were reported by the French group [19], here we offer a more complete and detailed analysis for such a luminescence as well as for the F-centre production efficiency. Although the above scheme essentially applies to most alkali halides, $\mathrm{NaCl}$ presents a number of advantages for our investigation. It presents a low efficiency for F-centre generation at low temperatures $\left(10^{5}-10^{6} \mathrm{eV} / \mathrm{F}\right.$-centre $)$ so that recombination is mostly radiative. This allows us to pick out more clearly the synergic effect between the thermal and excitation spikes.

\section{Swift-ion bombardment: coupled kinetics for the exciton and thermal spikes}

The effect of ion bombardment can be described by the accumulation of independent impact events. For every impact a linear region of damage is generated along the trajectory (damage track). The sequence of events following impact is as follows: electrons are, first, ionized into conduction band states through Coulomb scattering with the incoming ion. During the first $10^{-14} \mathrm{~s}$ these so-called delta electrons collide among themselves and reach certain thermalized energy distributions and certain radial profiles [24]. Up to then, the atomic lattice is unmodified. At later times the electron-phonon interaction sets-in and the energy is transferred to the atoms until a rough thermal equilibrium between electrons and atoms is attained. Finally, a cooling stage occurs and the room temperature of the system is, again, reached.

As a consequence of those events, a thermal spike is already established at around $10^{-12} \mathrm{~s}$ after the ion impact together with an excitation spike constituted by the thermalized cloud of electron and hole pairs. It is, moreover, assumed, in accordance with the available evidence, that these pairs become self-trapped in the lattice and constitute an exciton spike. The structure of the self-trapped exciton (STE) is that of an electron bound to a self-trapped hole (molecular $\mathrm{X}_{2}^{-}$ion). The STE undergoes radiative recombination emitting characteristic emission bands from the lowest excited level triplet state ${ }^{3} \Sigma_{u}^{+}$( $\pi$ emission) and from the singlet state ${ }^{1} \Sigma_{u}^{+}$( $\sigma$ emission). Also, the STE experiences non-radiative recombination that leads to molecular dissociation and point defect creation (Frenkel F-H or V-I pairs). Therefore, the recombination rate for the concentration $N_{\mathrm{X}}$ of STE can be described by a simple equation

$$
\frac{\mathrm{d} N_{\mathrm{X}}}{\mathrm{d} t}=-N_{\mathrm{X}}\left\{\frac{1}{\tau}+v_{0} \exp \left(-E / k_{\mathrm{B}} T\right)\right\}
$$

that includes both, radiative and non-radiative terms. $\tau$ is the radiative lifetime, $v_{0}$ an effective vibrational frequency and $E$ an energy barrier separating the bound and unbound regions of the excited state of the STE $[10,11]$.

The kinetics of the thermal spike has been analysed in detail by Toulemonde and co-workers by means of two coupled equations describing the electron and phonon dynamics, respectively $[6,7]$. For convenience, it is useful to apply the analytical approximation proposed by Szenes to the thermal spike kinetics [8]. One represents the spike by a Gaussian radial profile with width $a(t)$. The origin of time $(t=0)$ is set at the point where the thermal spike has reached equilibrium with the lattice (phonon bath). The dependence of the profile on penetration depth $z$ is determined by the corresponding value of the stopping power $S_{\mathrm{e}}(z)$. Using cylindrical coordinates with the $Z$ axis along the ion trajectory, the radial and depth profile for the temperature enhancement in the thermal spike is

$$
\Delta T(z, r, t)=\left(\frac{a_{0}}{a(t)}\right)^{2} \frac{g S_{\mathrm{e}}(z)}{\pi a_{0}^{2} \rho C} \exp \left\{-\frac{r^{2}}{a^{2}(t)}\right\} .
$$

The time evolution $a(t)$, with $a(0)=a_{0}$, is exclusively derived from the heat transport equation and $g=1-E_{\mathrm{G}} / I$, the fraction of ion energy deposited as heat with $E_{\mathrm{G}}$ the solid band gap and $I$ an effective ionization energy $\left(I \approx 2-3 E_{\mathrm{G}}\right)$. The parameters $\rho$ and $C$ represent the sample density and heat capacity, respectively.

For the associated exciton spike we assume that initially $(t=0)$ it is spatially correlated to the thermal spike profile, therefore,

$$
N_{\mathrm{X}}(z, r, 0)=\frac{1}{\pi a_{0}^{2}} N_{\mathrm{X}}(z, 0,0)\left\{\exp \left(-\frac{r^{2}}{a_{0}^{2}}\right)\right\}
$$

with

$$
N_{\mathrm{X}}(z, 0,0)=\eta S_{\mathrm{e}}(z) / I
$$


An efficiency factor $\eta$ has been introduced in expression (4). since absolute measurements [20] of the luminescence yield indicate that only a small fraction $(\sim 1 \%)$ of the excitons produced by x-ray irradiation of $\mathrm{NaCl}$ contribute to $\pi$-emission at low irradiation temperatures. Following the French group, an appropriate (measured) value for such an efficiency is $\eta=0.4 \%$. Note that we use the same value independently of the irradiation source (either $\mathrm{x}$-rays or swift ions). This assumption yields a reasonable agreement with experiment, although it would require further analysis. Possible exciton diffusion has been neglected.

\section{Model simulations for the exciton dynamics in $\mathrm{NaCl}$}

Our simulations involve the solution of equation (1), assuming the time evolution of the thermal spike temperature determined by equation (2) and the exciton profile (3). This will provide the local exciton concentration $N_{\mathrm{X}}(r, z, t)$ as a function of time. As a first example of our simulations, figure 1 illustrates the time evolution of the peak concentration of excitons, defects and luminescent centres in addition to the temperature at the central point of the spike for a $929 \mathrm{MeV} \mathrm{Xe}$ ion impinging on $\mathrm{NaCl}$ at $80 \mathrm{~K}$. Note that at this temperature the last (non-radiative) term in equation (1) dominates the radiative term ( $\tau$ was assumed to be $0.3 \mathrm{~ms}$ ). For the initial spike radius we have used a value $a_{0}=12 \mathrm{~nm}$ that is in between the values previously used by Protin $[19,20]$ to optimize the fit to the experimental luminescence yield values (see section 4.1). The thermal barrier $E$ for dissociation in equation (1) has been taken as $0.1 \mathrm{eV}[16,17,19,20]$. The values plotted in figure 1 are obtained by fixing $r=0$ and $z=0$ and leaving time $t$ as the only remaining variable. In accordance with Szenes's approach, the origin of time $(t=0)$ is set once the electronic and ionic temperatures at the spike are equilibrated and reach a maximum value. With these values, one observes that most defects are formed in the first $100 \mathrm{ps}$ after the origin of time when the temperature in the track centre is above $600 \mathrm{~K}$. Due to the long lifetime of the excitons the luminescence signal appears much later in the $\mu$ s range. In figure 2 radial profiles of spike temperature, excitons, defects and luminescent centres (photons) are given at different times after ion impact. It is clear from figures 1 and 2 that the kinetics and profiles of the two coupled spikes are quite different. The temperature evolution stems from the heat equation, therefore, the profile remains Gaussian at any given time. On the other hand, the exciton concentration rapidly drops via non-radiative decay in the region of the spike where the temperature is sufficiently high, i.e. in the track centre. As a consequence of this decay, defect production takes place and its corresponding radial profile follows the temperature evolution, being maximum at the track centre. Finally, at much longer times radiative exciton decay occurs from the remaining excitons, which act as luminescent centres whose radial distribution presents a maximum at $r \sim 18 \mathrm{~nm}$. Time-resolved irradiation experiments should be carried out to check these predictions.

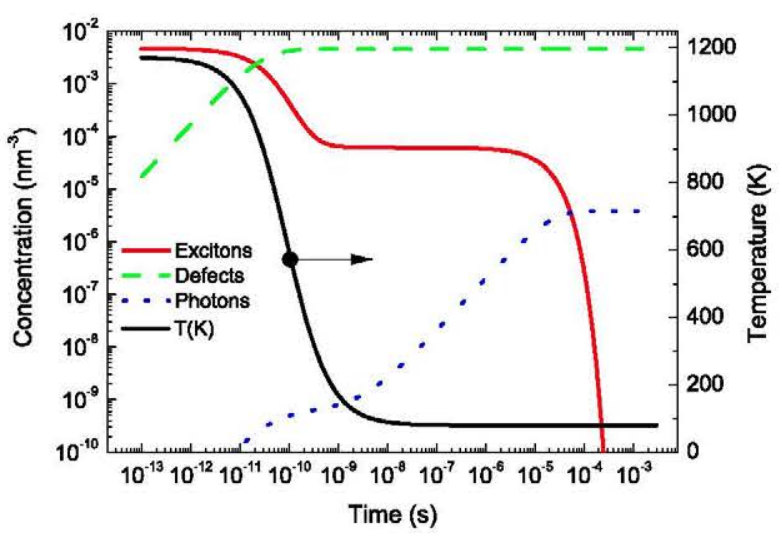

Figure 1. Time evolution of the concentration of excitons, defects and luminescent centres (photons) at the central point of the trajectory of a $\mathrm{Xe}$ ion impinging a $\mathrm{NaCl}$ surface with an energy of $929 \mathrm{MeV}$. The sample is assumed to be at $80 \mathrm{~K}$. The origin of time is taken as the moment at which both the temperature and the exciton concentration reach a maximum at the centre of the ion trajectory. For comparison the temperature in the track centre is given as a function of time.

\section{Simulation of experimental data}

Experimental data have been obtained for $\mathrm{NaCl}$ crystals irradiated in GANIL (Caen) with Zn at $735 \mathrm{MeV}$ and Xe at $929 \mathrm{MeV}$, whose electronic stopping curves $S_{\mathrm{e}}(z)$, determined by the SRIM 2000 code, are shown in figure 3. Note that both ions are rather penetrating $>130 \mu \mathrm{m}$ and $>70 \mu \mathrm{m}$, respectively. Due to the high values of electronic stopping power, almost linear tracks are formed with both ions, mainly by means of electronic excitation mechanisms. Samples were irradiated and measured in situ at the GANIL laboratory in the $15-300 \mathrm{~K}$ temperature range. They were covered by a copper mask with a central hole of $0.28 \mathrm{~cm}^{2}$ to assure uniform temperature. Ion fluxes were in the range $10^{4}-10^{10} \mathrm{~cm}^{-2} \mathrm{~s}^{-1}$. X-ray irradiations could be alternatively performed in the chamber. More technical details can be consulted in [20,21].

\subsection{Luminescence emission yield}

The $\pi$ emission yields produced by these ions at several temperatures above $15-20 \mathrm{~K}$ are displayed in figure 4 , together with the corresponding data for x-ray irradiation. All curves show the same trend with temperature and even present similar absolute yield values except for a significant decrease for Xe irradiations. In the range from $15 \mathrm{~K}$ to around $70 \mathrm{~K}$ the yield keeps essentially constant and corresponds to full radiative emission, i.e. at this low temperature the non-radiative channel is not dominant and subsequently the defect production rate is very low. At increasing temperature the luminescence yield approaches zero. This is due to the competing non-radiative exciton decay channel that turns out to be thermally activated. The luminescence yield in the case of Xe irradiation is lower than in the other cases due to the higher energy density deposited by these ions, which enable non-radiative decay from very low sample temperature.

To simulate the experimental data presented in figure 4 , i.e. the overall luminescence yield (total number of emitted 

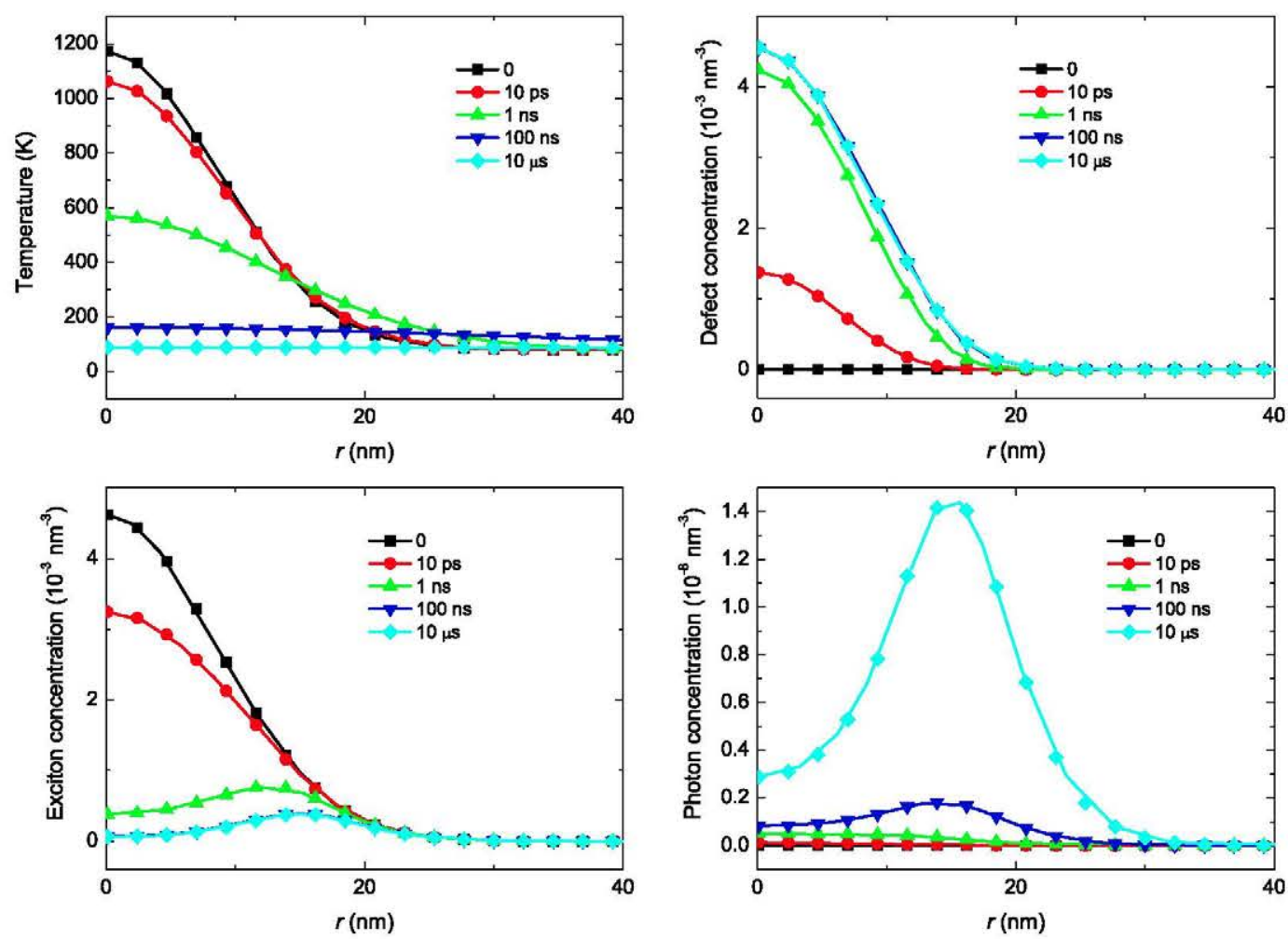

Figure 2. Profiles of temperature, exciton concentration, defect concentration and luminescent centres (photon emitters) at the surface of a $\mathrm{NaCl}$ sample kept at $80 \mathrm{~K}$ and irradiated with $929 \mathrm{MeV}$ Xe ions.

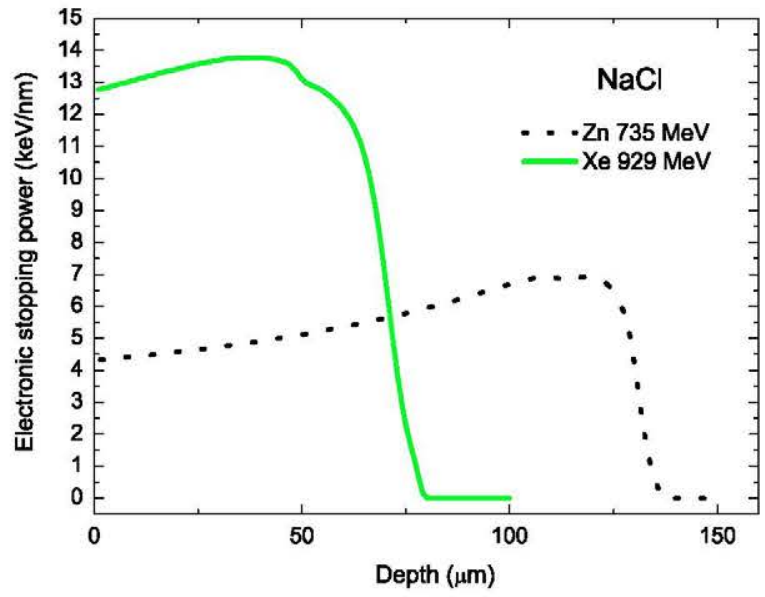

Figure 3. Electronic stopping power as a function of depth for the ions indicated in the legend impinging on $\mathrm{NaCl}$.

photons per impact), one should, first, compute the radial profiles of photons (see figure 2) emitted within a layer of unit thickness at depth $z$. They are obtained from the non-radiative channel of equation (1), as follows

$$
Y_{\mathrm{L}}(r, z)=\int_{0}^{\infty} \mathrm{d} t \frac{N_{\mathrm{X}}(r, z, t)}{\tau} .
$$

Then, for the total number of photons one has to integrate over $r$ and $z$,

$Y=\int_{0}^{L} Y_{\mathrm{L}}(z) \mathrm{d} z=\int_{0}^{\infty} 2 \pi r \mathrm{~d} r \int_{0}^{\infty} \mathrm{d} t \frac{N_{\mathrm{X}}(r, z, t)}{\tau} \int_{0}^{L} \mathrm{~d} z(6)$

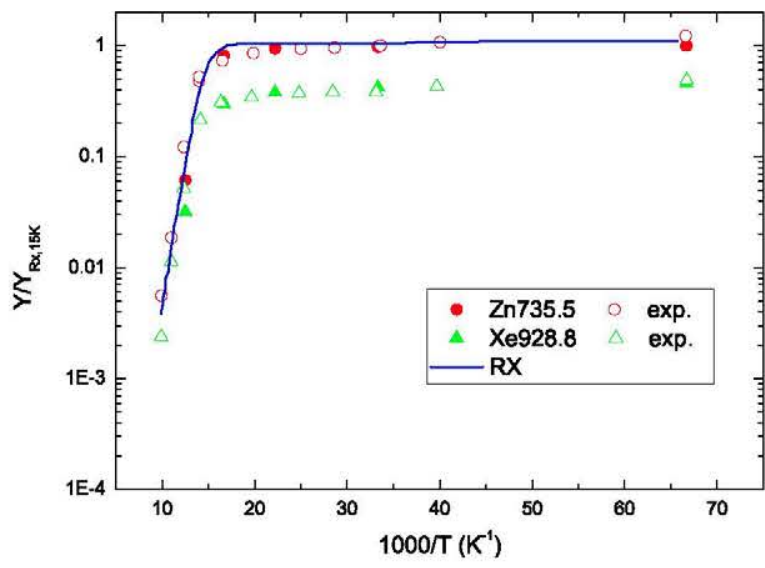

Figure 4. Experimental data (open symbols) corresponding to luminescence yield obtained by $\mathrm{NaCl}$ irradiation with the ion indicated in the legend $[19,20]$. The (blue) line corresponds to irradiations with $\mathrm{x}$-rays. The solid symbols were obtained as an application of the two-spike model described in the paper.

where $Y_{\mathrm{L}}(z)$ stands for the number of photons emitted within a unit thickness layer at depth $z$. The final step of the simulation is to integrate that differential yield over the whole depth range $L$ of the trajectory, i.e. from the initial value at the surface, $S_{\mathrm{e}}(z=0)$, to the value at which $S_{\mathrm{e}}(z=L)$ becomes negligible. For practical purposes we take $L$ such that $S_{\mathrm{e}}$ is half its maximum value (see figure 3 ).

Using this formalism, the results of the best-fit simulations are represented by solid symbols in figure 4 together with the experimental data (open symbols). The 
best fit to experiment was achieved for $a_{0}=12 \mathrm{~nm}$ that is in between the values previously used $[19,20]$. The calculated points reproduce fairly well the experimental curves. Note that the only free parameter was the initial width of the temperature and exciton Gaussian distributions. The agreement is remarkable because it is obtained in a broad range of temperature for irradiations with two different ions. From the employed model we can explain the experimental results. At low temperatures most of the available excitons undergo radiative decay. Only irradiations with energetic Xe ions promote significant non-radiative decay events that result in a lower luminescence yield. This is possible because part of the energy deposited in the track contributes to activate the non-radiative channel. At around $70 \mathrm{~K}$ the non-radiative channel starts to dominate, being the major exciton decay channel at around $100 \mathrm{~K}$. Figure 2 shows the radial profiles at an intermediate irradiation temperature $(80 \mathrm{~K})$ to illustrate the temperature role on exciton decay. The kinetics of exciton decay depend on a balance between the radiative channel and the thermally activated non-radiative channel.

\subsection{F-centre growth curves (production efficiency)}

In order to check the validity and robustness of our model we applied it to account for the defect production (F-centres). Defect production takes place via the non-radiative exciton decay and therefore constitutes the alternative channel to luminescence emission. This is, in fact, the most relevant and novel contribution of our theoretical approach. The initial F-centre growth curves measured in GANIL for Xe irradiation on samples at two different temperatures $(10$ and $80 \mathrm{~K})$ are plotted in figure 5. The temperature range below around $100 \mathrm{~K}$ is the most meaningful since aggregation processes are minimized and thus, F-centres account for all point defects generated by electronic excitation upon irradiation (note that we are not discussing defect formation by elastic collisions). The F-centre growth curves must be measured at low enough fluences $\left(10^{9}-10^{11} \mathrm{~cm}^{-2}\right)$ where ion impacts are uncorrelated, i.e. the damaged areas are not overlapped. Track overlapping may lead to enhanced annealing effects that are not easily taken into account. Finally, we should realize that the measurements refer to the relaxed or uncorrelated population of the generated F-centres after the primary Frenkel pairs are separated and become uncorrelated [18].

For the theoretical simulations of those results we follow the same approach as for the luminescence yield but now referring to the non-radiative decay term in equation (1). For the radial profile of the generated defect concentration one has

$$
n_{\mathrm{F}}(r, z)=\int_{0}^{\infty} \mathrm{d} t v_{0} N_{\mathrm{X}}(r, t, z) \exp \left\{-\frac{E}{k_{\mathrm{B}} T(r, z, t)}\right\}
$$

A simulated profile of the generated concentration of F-centres is shown in figure 2 for comparison with the spike profiles. The total concentration over the whole sample thickness $L$ (as measured in an optical absorption experiment) is given by

$$
N_{\mathrm{F}}=\int_{0}^{L} \mathrm{~d} z \int_{0}^{\infty} \mathrm{d} r n_{\mathrm{F}}(r, z)
$$

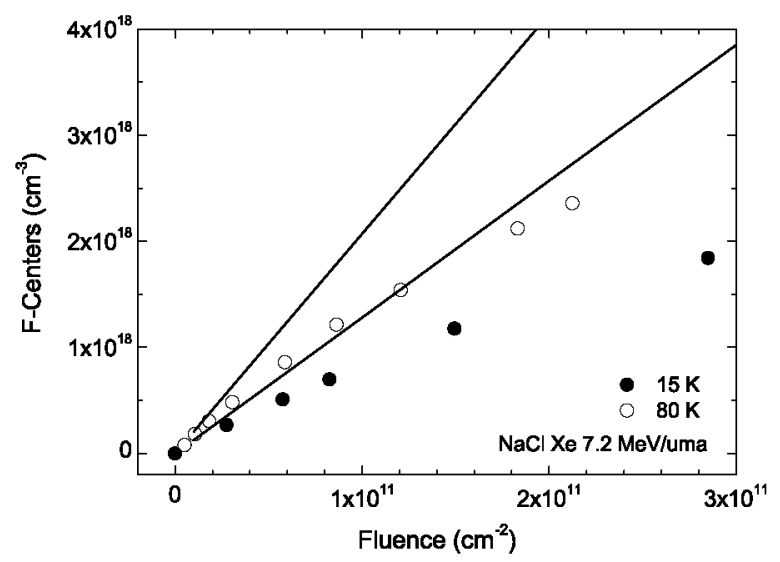

Figure 5. Concentration of F-centres as a function of fluence for irradiation with $\mathrm{Xe}$ ions at $929 \mathrm{MeV}$ at the temperatures indicated in the legend [20]. The lines were obtained from the two-spike model with the parameters used to fit the luminescence yields. Note that the initial defect generation rate is fairly well reproduced (low fluences).

The theoretical F-centre growth curves are included in figure 5 for comparison with the experimental values at 15 and $80 \mathrm{~K}$. We have used the same model parameters as those used to fit and optimize the luminescence data. The simulated lines represent the initial linear stage of the overall growth curves, i.e. the low fluence region. In view of the relative simplicity and phenomenological nature of the model a precise agreement between experiment and theory is not expected. However, it is remarkable how the model is able to reproduce fairly well, both the luminescence yield and the initial F-centre concentration kinetics, with the same set of realistic parameters. For higher fluences the predictions from the model clearly overestimate the F-centre production observed experimentally. Aggregation and annealing of defects, which are not considered in the model, may play a significant role.

\section{Summary and conclusions}

We have applied a non-radiative exciton decay model (well documented for alkali halides) coupled to the thermal spike approach (well established for many dielectrics) to understand, both the luminescence emission yield and the rate of F-centre growth under swift ion-beam irradiation. The data were obtained by the GANIL group $[19,20]$. Our work focuses on low temperature irradiations $(<100 \mathrm{~K})$ so that one can neglect defect aggregation processes. The reasonable agreement between theory and experiment for a crystal like $\mathrm{NaCl}$, where exciton dynamics is well ascertained, provides quantitative support to the model previously used for $\mathrm{LiNbO}_{3}$. It is expected that the synergy between the thermal and excitation spikes may play an essential role in understanding damage mechanisms by swift ions in other dielectric materials.

\section{Acknowledgments}

We express our deep gratitude to $\mathrm{M}$ Toulemonde, $\mathrm{E}$ Balanzat and other members of GANIL for the data and information 
provided on the experiments. We also appreciate useful discussions with them on the subject of this work.

Nastase M, Mayer J W and Hirvonen J H 1996 Ion-Solid Interactions: Fundamentals and Applications (Cambridge: Cambridge University Press)

Ziegler J F 1992 Ion Implantation Technology (Amsterdam: North-Holland)

Itoh $\mathrm{N}$ and Stoneham M 2001 Materials Modification by Electronic Excitations (Cambridge: Cambridge University Press)

Fleischer R L, Price P B and Walker R M 1965 J. Appl. Phys. 363645

Lesueur D and Dunlop A 1993 Radiat. Eff. Defects Solids 126163

Toulemonde M, Dufour Ch, Meftah A and Paumier E 2000 Nucl. Instrum. Methods Phys. Res. B 166903

Meftah A, Constantini J M, Khalfaoui N, Boudjadar S, Stoquert J P, Studer F and Toulemonde M 2005 Nucl. Instrum. Methods Phys. Res. B 237563

Szenes G 1999 Phys. Rev. B 603140

Itoh N 1998 Nucl. Instrum. Methods B 146362

Agulló-López F, Méndez A, García G, Olivares J and Cabrera J M 2006 Phys. Rev. B 74174109
Rivera A, Méndez A, García G, Olivares J, Cabrera J M and Agulló-López F 2008 J. Lumin. 128703

Itoh N, Duffy D M, Khakshoury S and Stoneham A M 2009 J. Phys.: Condens. Matter. 21474205

Williams R T, Bradford J N and Faust W L 1978 Phys. Rev. B 187038

Agullo-López F, Catlow C R and Townsend P D 1984 Point Defects in Materials (London: Academic)

Williams R T and Song K S 1996 Self-trapped Excitons 2nd edn (Berlin: Springer)

Sonder E 1972 Phys. Rev. B 53259

Sonder E 1975 Phys. Rev. B 121516

Agull6-Lopez F and Townsend P D 1980 Solid State Commun. 33449

Balanzat E, Bouffard S, Cassimi A, Doorhye E, Protin L, Grandin J P, Doualan J L and Margerie J 1994 Nucl. Instrum. Methods Phys. Res. B 91134

Protin L 1994 PhD Thesis University of Caen

Protin L et al 1995 Radiat. Eff. Defects Solids 136287

Schwartz K, Trautmann C, El-Said A S, Neumann R, Toulemonde M and Knolle W 2004 Phys. Rev. B 70184104

Enculescu M, Schwartz K, Trautmann C and Toulemonde M 2005 Nucl. Instrum. Methods Phys. Res. B 229397

Waligorski M P R, Hamm R N and Katz R 1986 Nucl. Tracks Radiat. Meas. 1139 